Rabaska

Revue d'ethnologie de l'Amérique française

\title{
Raynald Bilodeau, l'ethnologue qui fait parler les décors intérieurs
}

\section{Raynald Bilodeau, an ethnologist who brings interior decoration to life}

\section{Bernard Genest et Claire Desmeules}

Volume 17, 2019

URI : https://id.erudit.org/iderudit/1066012ar

DOI : https://doi.org/10.7202/1066012ar

Aller au sommaire du numéro

Éditeur(s)

Société québécoise d'ethnologie

ISSN

1703-7433 (imprimé)

1916-7350 (numérique)

Découvrir la revue

Citer cet article

Genest, B. \& Desmeules, C. (2019). Raynald Bilodeau, l'ethnologue qui fait parler les décors intérieurs. Rabaska, 17, 133-152. https://doi.org/10.7202/1066012ar
Résumé de l'article

Raynald Bilodeau, technicien en restauration, est connu et reconnu comme spécialiste des décors de bâtiments historiques, particulièrement au niveau des revêtements extérieurs et intérieurs, et plus spécialement encore des papiers peints. Au cours de sa longue carrière, par intérêt personnel aussi bien que professionnel, il a constitué une collection unique et exceptionnelle de papiers peints datant des années 1800 à 1930. La collection compte 1200 modèles différents de papiers peints prélevés sur plus de 200 sites, tous situés au Québec. Un outil de référence incontournable pour la restauration et l'interprétation des intérieurs anciens et pour l'histoire de l'habitat domestique québécois. L'article présente le parcours professionnel du spécialiste qui, en 2006, a légué sa collection à son employeur, Parcs Canada. 


\title{
Terrains
}

Raynald Bilodeau, l'ethnologue qui fait parler les décors intérieurs ${ }^{1}$

\author{
Bernard Genest \\ Société québécoise d'ethnologie \\ avec la collaboration de Claire DesmeUles \\ Parcs Canada, Québec
}

Raynald Bilodeau est né le 12 août 1951 à Québec. Il est le fils de Gaston Bilodeau (1919-1987), ébéniste, et de Thérèse Bélanger. Ses deux grandspères, tant Bélanger que Bilodeau, étaient menuisiers. Technicien en restauration de mobilier à Parcs Canada pendant 30 ans, Raynald dit devoir beaucoup à la tradition familiale du travail du bois. Enfant, il accompagnait son père sur les chantiers de construction, l'assistant dans de menus travaux, s'intéressant à tout, observant faits et gestes, faisant montre d'une sensibilité pour les arts, dessinant partout et tout le temps, et reproduisant en miniature, maisons, voitures et objets de la vie courante. Ces années au sein d'une famille de neuf enfants ont, dit-il, été déterminantes dans l'exercice de sa profession. Après avoir envisagé d'entreprendre une carrière en arts et communications graphiques - domaine dans lequel il a travaillé pendant quelques années - il s'est d'abord dirigé en techniques policières au cégep Garneau, pour finalement réaliser que cette vocation «n'était pas pour lui ». C'est à ce moment qu'il découvre que l'Université Laval offrait un programme d'études en ethnologie portant sur les traditions orales, les coutumes et la culture matérielle. Il s'inscrit sans savoir précisément où cela allait le conduire. En 1981, après avoir complété ses études, il rejoint l'équipe de restauration de Parcs Canada. Au cours des ans, il développe une expertise pointue en matière de mobilier et de finis intérieurs et extérieurs des bâtiments anciens. Dès son entrée à Parcs Canada, il commence à monter, de sa propre initiative et par intérêt personnel, une collection unique et exceptionnelle de papiers peints prélevés sur le terrain. L'exercice s'étendra sur une trentaine d'années et la collection sera léguée à Parcs Canada juste avant qu'il ne prenne sa retraite.

1. Ce sous-titre s'inspire d'un article paru dans Le Soleil du samedi 11 novembre 2014, sous la signature de Michèle Laferrière : «Un ethnologue fait parler le papier peint ». 


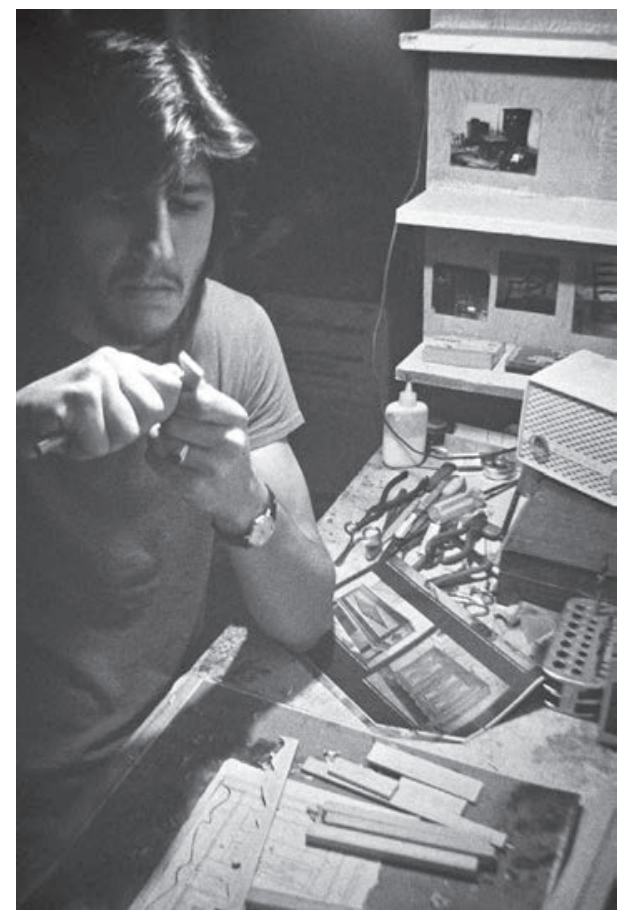

Raynald Bilodeau

Photo : Lise Cardinal, 2019

\section{Le travail du bois, une tradition familiale}

Fils d'ébéniste et petit-fils de menuisiers charpentiers, Raynald a été élevé dans un milieu d'artisans qui valorisaient le travail « bien fait» comme la plupart des artisans de cette époque. Déjà, à l'âge de 8 ans, il suivait son père sur les chantiers de construction, dont celui de Parc Falaise, lotissement réalisé à partir de 1948 dans l'ancienne ville de Sillery, un ensemble résidentiel qui a fait l'objet de maints éloges pour son caractère novateur. Créé à l'initiative d'Eugène Chalifour, conseiller municipal et homme d'affaires, « Parc Falaise met à profit les dernières tendances en matière d'architecture résidentielle et d'aménagement urbain, privilégiant le bungalow comme modèle d'habitation ». Les entrepreneurs, les frères Couillard de Lauzon, avaient fait appel à Gaston Bilodeau pour l'exécution des travaux de finition. Spécialiste d'ébénisterie fine, boiseries, moulures, appliques, escaliers tournants et autres éléments décoratifs des intérieurs bourgeois, Gaston Bilodeau était aussi constructeur de bateaux sur les chantiers de construction navale de Sillery. Autant d'occasions pour l'enfant qui suivait son père partout d'aiguiser sa curiosité, d'observer les choses qui l'entouraient, les gestes des artisans qu'il 
côtoyait. C'est ainsi qu'il sut très tôt distinguer l'usage et la fonction de chacun des instruments qui se trouvaient dans le coffre d'outils de son père et que, dans ses jeux, il ne songeait qu'à entreprendre des projets de construction, comme des maquettes de bateaux et de voitures, ou des villages miniatures comme celui qu'il avait fait naître dans le sous-sol de la résidence familiale : « Un village de carton, avec des maisons, mais aussi des bâtiments publics, des restaurants et des garages $»$. De cette époque, Raynald a conservé précieusement le coffre à outils de son père, objet culte qui lui rappelle l'admiration qu'il avait pour le savoir-faire de ce dernier. Plusieurs des outils contenus dans ce coffre sont de la main même de l'ébéniste, souvent inventés ou « patentés » par lui pour répondre à un besoin précis. La plupart des manches sont faits de bois précieux, chêne, acajou, teck, taillés à même les retailles de bois provenant des chantiers où l'artisan travaillait. Tous ces outils portent la marque discrète de l'ébéniste, symbole d'appartenance indispensable sur les chantiers de construction. Cette marque consistait en deux traits parallèles profondément creusés dans le matériau, bois ou métal. Toujours harmonieuse, jamais disgracieuse, elle était appliquée directement sur le manche d'une

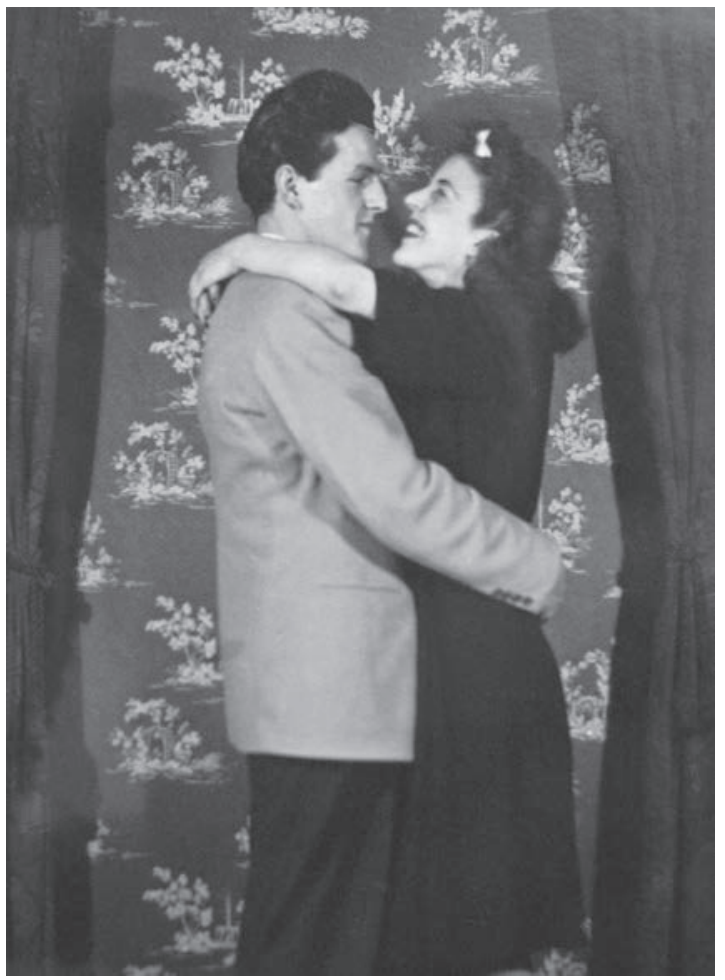

Gaston Bilodeau et Thérèse Bélanger [les parents de Raynald], devant un papier peint de la fin des années 1930 
scie ou d'un marteau ou, encore, sur la douille de métal fixant la lame au manche d'un ciseau ou d'une gouge. Raynald rappelle que son père était un homme méticuleux « dans son travail comme sur sa personne». Il travaillait toujours " en chemise et en cravate », enfilant un survêtement au moment de se mettre à l'ouvrage.

\section{Le parcours scolaire}

Enfant, Raynald fréquente les écoles primaires Maria-Goretti et SaintDominique-Savio de Les Saules. Les Bilodeau étaient de Limoilou, mais Gaston Bilodeau avait profité d'un programme de prêts hypothécaires pour construire sa maison dans une coopérative d'habitations dans la nouvelle petite municipalité de Sainte-Monique-des-Saules. Raynald fait son secondaire à Loretteville, puis travaille un an et demi au ministère de la Voirie à des travaux d'arpentage, avant de poursuivre ses études au cégep Garneau, boulevard de l'Entente, pour y suivre une formation en techniques policières. C'est à cette époque qu'il développe des aptitudes en photographie, tant et si bien qu'on le nomme responsable du laboratoire photo du collège. Six mois avant la fin de son parcours - qui devait se terminer à l'École nationale de police de Nicolet - le jeune homme réalise que cette carrière n'est pas faite pour lui. Il s'informe et découvre que l'Université Laval donne une formation en Arts et traditions populaires, un champ d'études qui rejoint ses intérêts. Il s'y inscrit en se disant « qu'il serait toujours temps d'aller voir ailleurs » si ça ne répondait pas à ses attentes, mais il réalise bien vite qu'il est dans son élément. À travers l'enseignement de ses professeurs (Jean-Claude Dupont, Jean Du Berger, Élie-Kongas-Maranda, Robert-Lionel Séguin, Jean Simard), il comprend que c'est l'ensemble du champ, tant immatériel que matériel, qui donne un sens à ses propres préoccupations pour les objets de culture matérielle et les pratiques traditionnelles. Tout en poursuivant ses études, il travaille chez Cartier Photo, rue Cartier, puis, pendant trois ans, comme technicien en arts graphiques pour le ministère de l'Agriculture.

\section{Le modélisme : prélude à une carrière}

Lorsque, enfant, Raynald s'amusait à fabriquer des maisons de carton et des modèles réduits de bateaux et d'automobiles, il était loin d'imaginer que ce passe-temps était un prélude à sa carrière de restaurateur. C'est pourtant de là que lui vient cette curiosité pour les objets du passé. Déjà, il s'interrogeait sur la façon dont les choses étaient faites, la nature des matériaux utilisés, bois, métaux, verre, céramique, et sur leur décor et leur fonction. Plus tard, il voudra tout savoir du mobilier ancien des XVIII ${ }^{e}$ et XIX ${ }^{\mathrm{e}}$ siècles. Il se documente en consultant des ouvrages spécialisés, mais aussi en faisant des recherches sur 
le terrain et en rencontrant des antiquaires. Il forme le projet de reproduire en miniature l'habitat et les objets du quotidien de nos ancêtres. Il fait plusieurs essais avant d'en arriver à un résultat qui le satisfasse. Il retire de cet exercice de précieuses connaissances quant à la propriété des matériaux et à la manière de les travailler. Il dira : « Je travaille lentement, mais je travaille juste et bien! » Il reproduit à l'échelle et selon les modes d'assemblage d'autrefois (tenons et mortaises chevillés, queues d'aronde, mi-bois) meubles et objets de la vie quotidienne. Il commence habituellement par exécuter un simple croquis, puis un dessin qu'il calque ensuite sur la pièce de bois ou de métal (ou autre matériau) dont il tirera chacun des morceaux de l'objet qu'il veut reproduire. Il utilise surtout le pin et l'érable à grain fin, des bois mous « qui se travaillent bien et qui se teignent bien ». Il façonne au tour et martèle le laiton, le cuivre et le fer pour en faire des chaudrons, des candélabres, des pentures et des serrures. D'une chose à l'autre, il est amené à monter ce qu'il appelle « ses collections », c'est-à-dire à constituer des intérieurs miniaturisés d'habitations traditionnelles avec tous les éléments qui s'y trouvent: meubles, instruments d'éclairage, objets usuels et décoratifs, revêtements des murs, des plafonds et du sol. C'est à l'échelle 1/6 qu'il reproduit dans les moindres détails ces intérieurs, telle cette salle de séjour d'une habitation du début du $\mathrm{XIX}^{\mathrm{e}}$ siècle avec son mobilier : table rustique, chaises et fauteuils à fonds cannés, vaisselier et buffet bas, berceau, tapis crochetés ou tressés, âtre servant au chauffage et à la cuisson des aliments. Il s'interroge sur les éléments de décor, finis d'époque et couleurs à la mode. C'est ce qui l'amène à s'intéresser aux papiers peints et à en introduire dans le décor de ses reconstitutions miniaturisées. Il réalise ainsi deux ou trois de ces « collections » qu'il expose dans des bibliothèques et des centres d'exposition. Ses maquettes attirent l'attention du public et des journalistes qui lui consacrent quelques articles dans des quotidiens. Celles-ci sont exposées plusieurs fois au Musée de la civilisation qui en fait finalement l'acquisition.

À la fin des années 1970, dans le cahier Perspective de fin de semaine du journal Le Soleil, Raynald apprend que la Société Saint-Jean-Baptiste de Québec souhaitait « rapatrier » la célèbre maquette Duberger dans son lieu d'origine. Il connaissait l'histoire de ce plan-relief de la ville de Québec réalisé par l'arpenteur géomètre Jean-Baptiste Duberger et l'ingénieur anglais John By. L'œuvre, faisant à l'origine 6,15 mètres de largeur et près de 10 mètres, était une minutieuse reconstitution en miniature de la ville de Québec dans les années 1806-1808. Expédiée à la cour d'Angleterre, elle est présentée au roi dans le cadre d'une demande de financement pour l'amélioration des fortifications de Québec. Ramenée au Québec en 1908 à l'occasion du Tricentenaire (mais amputée de la moitié de sa longueur), elle prendra ensuite 
la direction d'Ottawa où elle ira, semble-t-il, encombrer les réserves du Musée canadien de la guerre. Apprenant que la Société Saint-Jean-Baptiste voulait la rapatrier, Raynald soumet sa candidature pour participer à sa restauration si elle devait effectivement revenir à Québec, mais ne reçoit aucune réponse de la part de l'organisme.

\section{«L'archéologue » des intérieurs anciens}

C'est Louise Bernard, chercheure à Parcs Canada, aussi chargée de cours en ethnologie à l'Université Laval, qui oriente Raynald vers la restauration. Il avait réalisé pour elle un travail de recherche dont l'orthographe laissait à désirer, mais dont le contenu avait impressionné la chargée de cours. Elle lui avait dit : «Raynald, tu fais beaucoup de fautes, mais je ne peux pas faire autrement que de te donner une bonne note »; et elle l'avait informé du même coup de l'ouverture d'un concours pour l'embauche de techniciens en restauration à Parcs Canada : «Il me semble, lui aurait-elle dit, que je te verrais bien là. » Cette remarque n'était pas tombée dans l'oreille d'un sourd. Compte tenu de ses expériences passées il avait, dit-il, « une longueur d'avance sur les autres candidats ». Effectivement, il passa le concours et fut engagé. Le hasard faisant bien les choses, un de ses premiers mandats fut, avec son collègue Jeannot Bélanger, restaurateur ébéniste, de rassembler, remonter et restaurer la maquette Duberger en vue de son installation au Parc d'Artillerie. Celle-ci, en effet, était bien revenue à Québec et confiée, non pas à la Société Saint-Jean-Baptiste, mais à Parcs Canada. À son retour

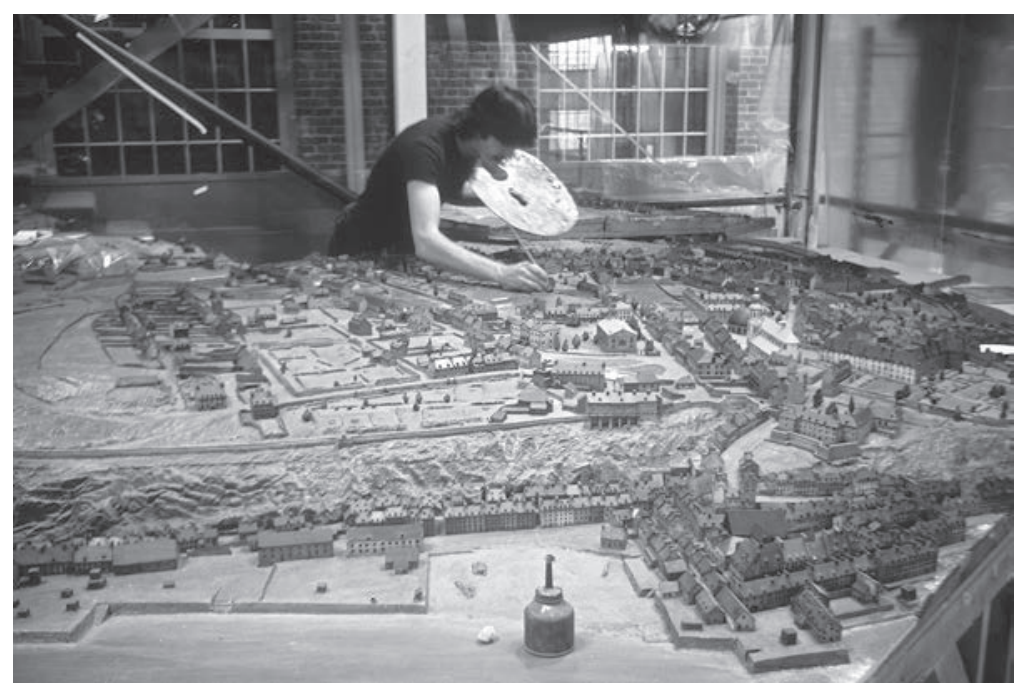

Raynald Bilodeau restaurant la maquette Duberger au Parc-de-l'Artillerie, en 1981 
d'Angleterre, la maquette avait fait l'objet d'une restauration majeure dans les années 1909-1910, puis à nouveau dans les années 1977-1978, mais, lors de son transport vers Québec, elle avait été découpée en neuf sections et, de ce fait, dépouillée de plusieurs des pièces qui la composaient. Le défi était de rassembler chacune des sections et de replacer au bon endroit les pièces manquantes - « un vrai casse-tête » dit-il - selon les indications de Tom Patridge, le chargé de mission du Musée canadien de la guerre. Il fallait aussi restaurer, repeindre et ajouter des éléments contextuels comme la présence de la rivière Saint-Charles et du fleuve Saint-Laurent, faire disparaître toutes les traces laissées par le réassemblage des sections et réinstaller le tout pour sa présentation au public. Et tout cela à l'intérieur d'un calendrier très serré. Raynald raconte que le matin même de l'inauguration, il était encore à exécuter des travaux de finition: «Je suis sorti par la porte arrière pour aller me changer, et suis rentré par une autre pour assister à l'ouverture. »

Selon sa définition de tâches à Parcs Canada, Raynald avait été engagé comme technicien en restauration de mobilier ancien, mais dans la pratique ses attributions étaient beaucoup plus étendues. Spécialiste des vernis et des couleurs d'origine, il devait aussi reproduire à l'identique des objets devant servir à reconstituer des intérieurs de bâtiments patrimoniaux, maisons, édifices, forteresses, casernes, prisons, autant de lieux historiques nationaux relevant du ministère du Patrimoine canadien. D'autres organismes fédéraux, tels le ministère des Travaux publics et le ministère de la Défense nationale, font aussi appel à son expertise. À l'instar des archéologues qui fouillent le sol pour mettre à jour les strates d'occupation d'un site pour en faire une lecture du mode de vie de communautés disparues, le chercheur devait s'appliquer à relever sur les extérieurs et les intérieurs de vieux édifices, séquence par séquence, toutes les traces laissées par les occupants au fil du temps et des générations, pour en tirer des informations relatives à l'usage des bâtiments comme à l'évolution des modes et des goûts. C'est ainsi qu'il notera qu'avant l'avènement de l'électricité, l'usage était de peindre en jaune des espaces peu éclairés comme, par exemple, une cage d'escalier. Ailleurs, l'analyse des échantillons lui permettra de faire connaître un épisode méconnu de l'histoire d'un bâtiment, tel un incendie. Effectuées en amont des travaux de restauration, ces opérations de curetage se sont révélées fort utiles pour l'ensemble des professionnels de la restauration, historiens, architectes, conservateurs et chargés d'interprétation.

Au cours de sa longue carrière, Raynald est intervenu de différentes façons (opérations de curetage et de nettoyage, application de vernis et de faux finis, pose de papiers peints, etc.) sur un très grand nombre de bâtiments historiques. Pour n'en citer que quelques-uns, mentionnons la maison George-Étienne 


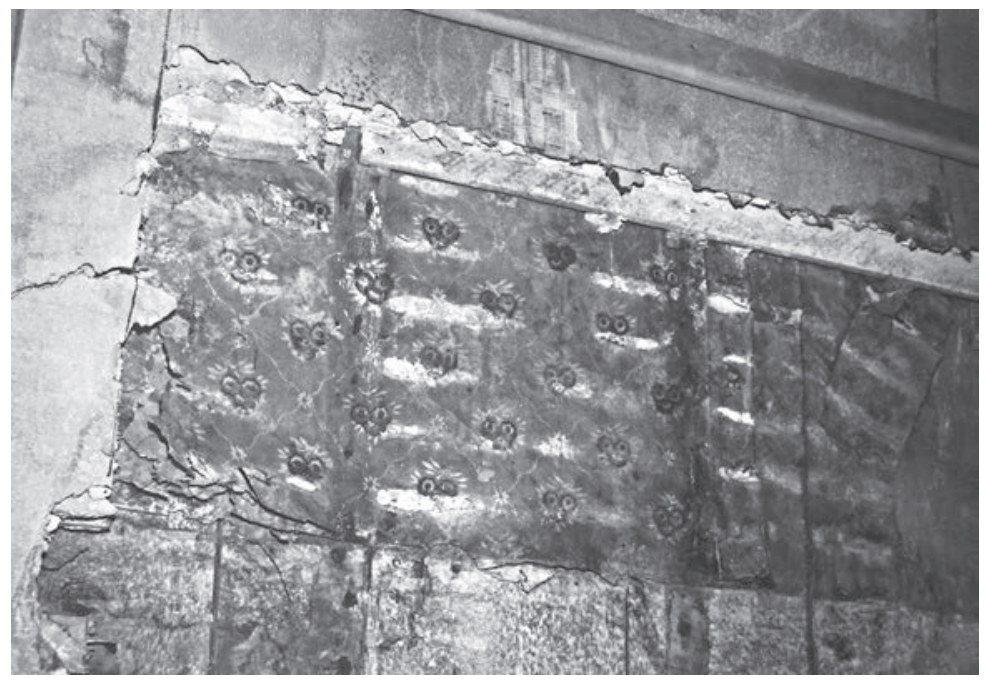

Papier peint collé directement sur un mur

Maison Jacques-DeGuise-dit-Flamand, Côte du Palais, Québec

Photo : Parcs Canada, vers 1975

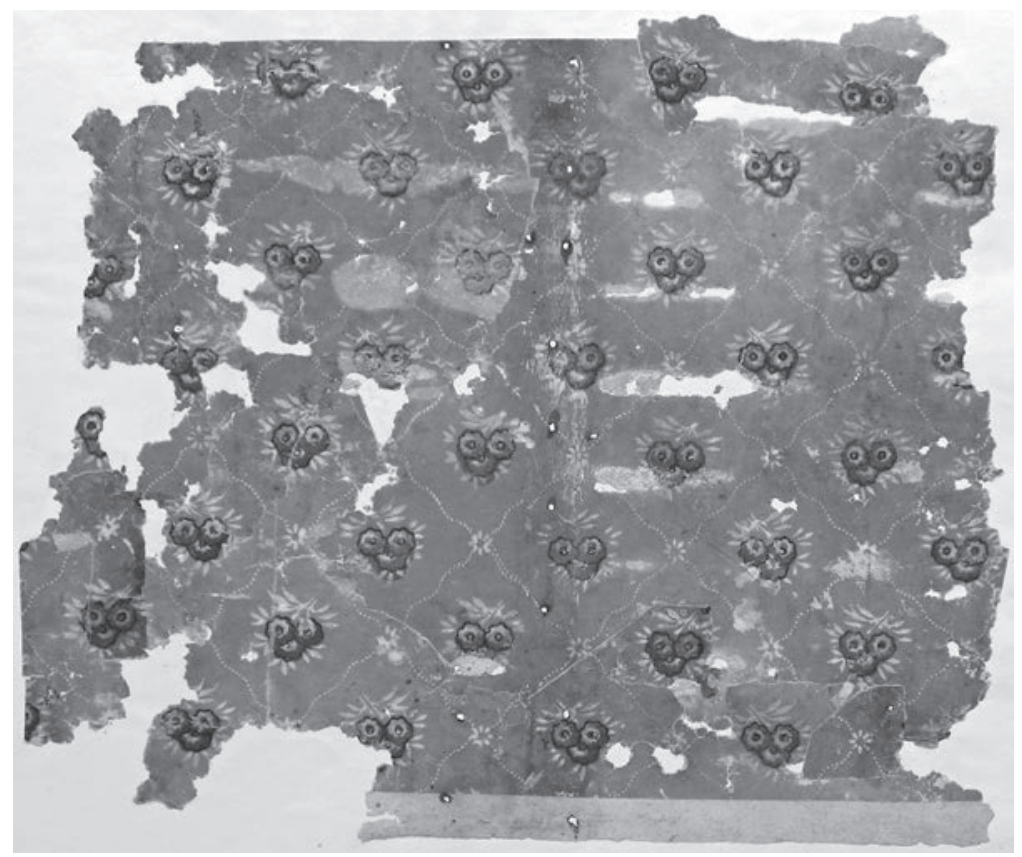

Fragment du papier peint précédent (vers 1800-1810)

Coll. Parcs Canada 


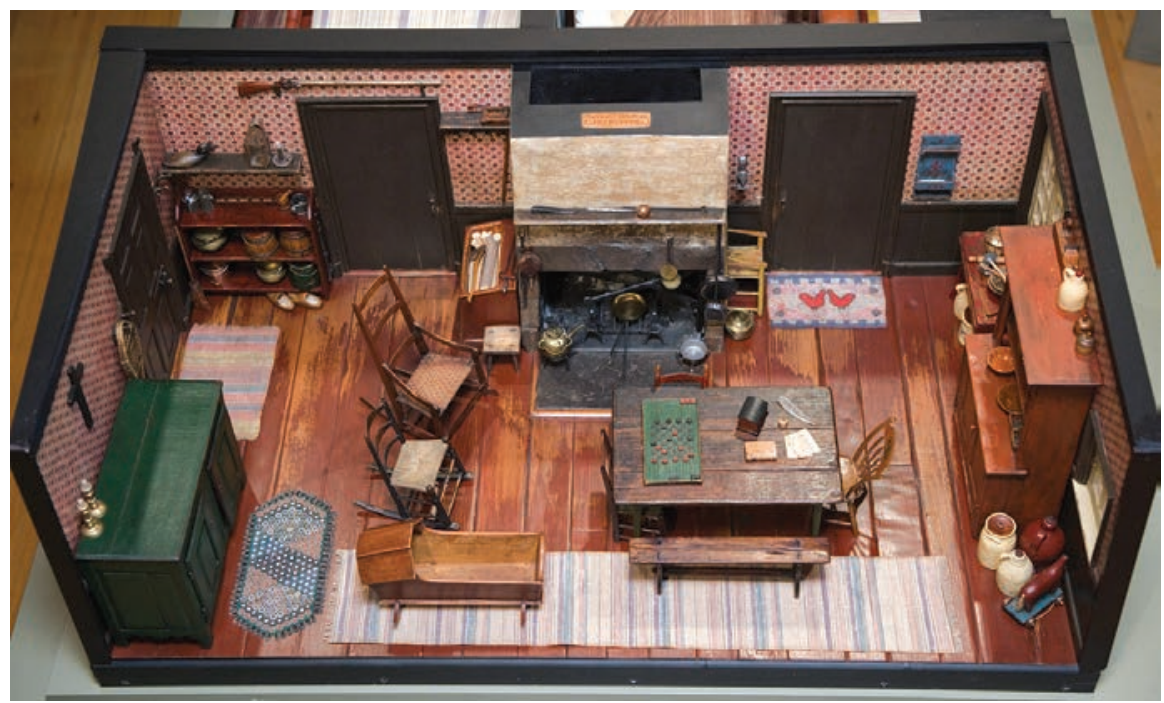

Maquette d'un intérieur québécois à l'échelle 1/6, réalisée par Raynald Bilodeau vers 1975

Collection du Musée de la civilisation Photo : Raynald Bilodeau

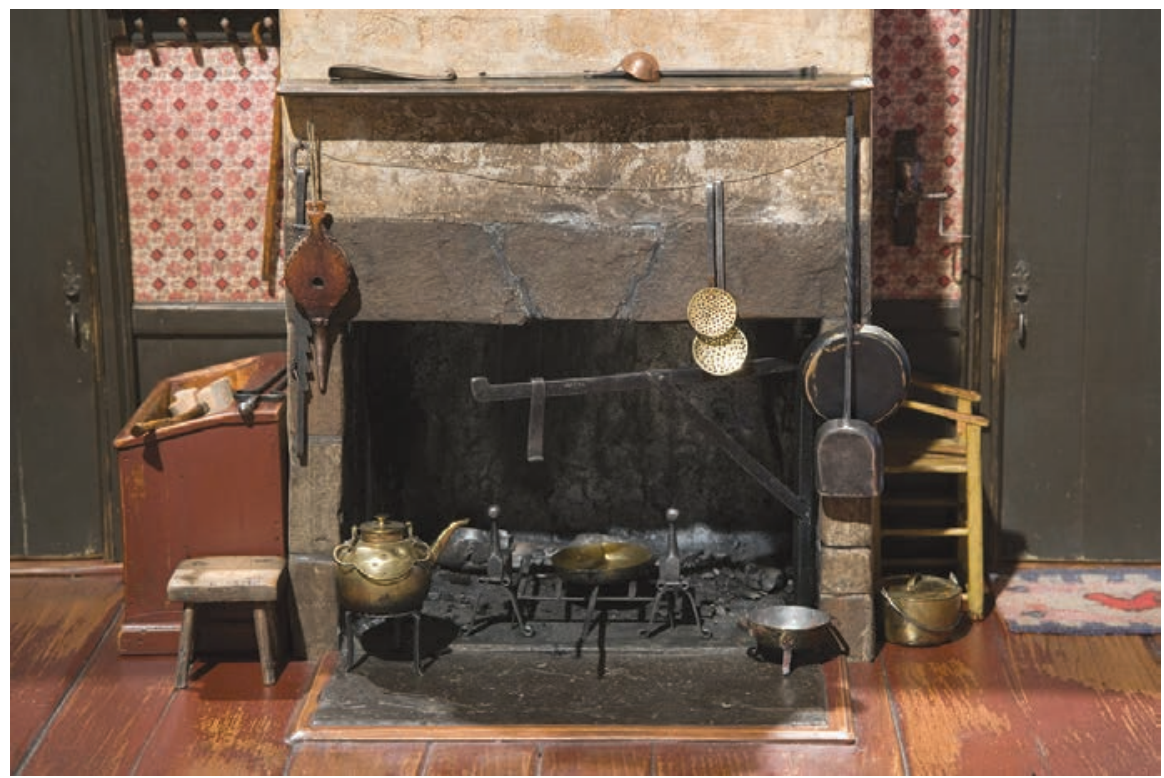

Détail de la photo précédente 


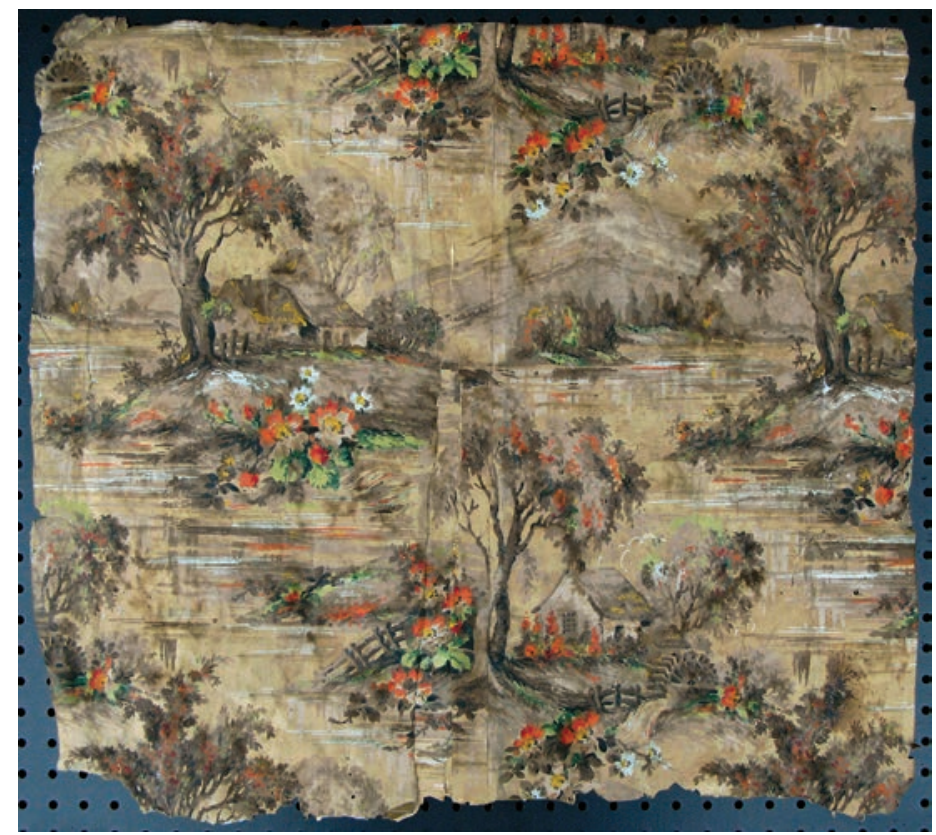

Détail de la dernière couche [la $\left.11^{\mathrm{e}}\right]$ de papier peint

Maison Louis-Fréchette, Lévis

Photo : Raynald Bilodeau

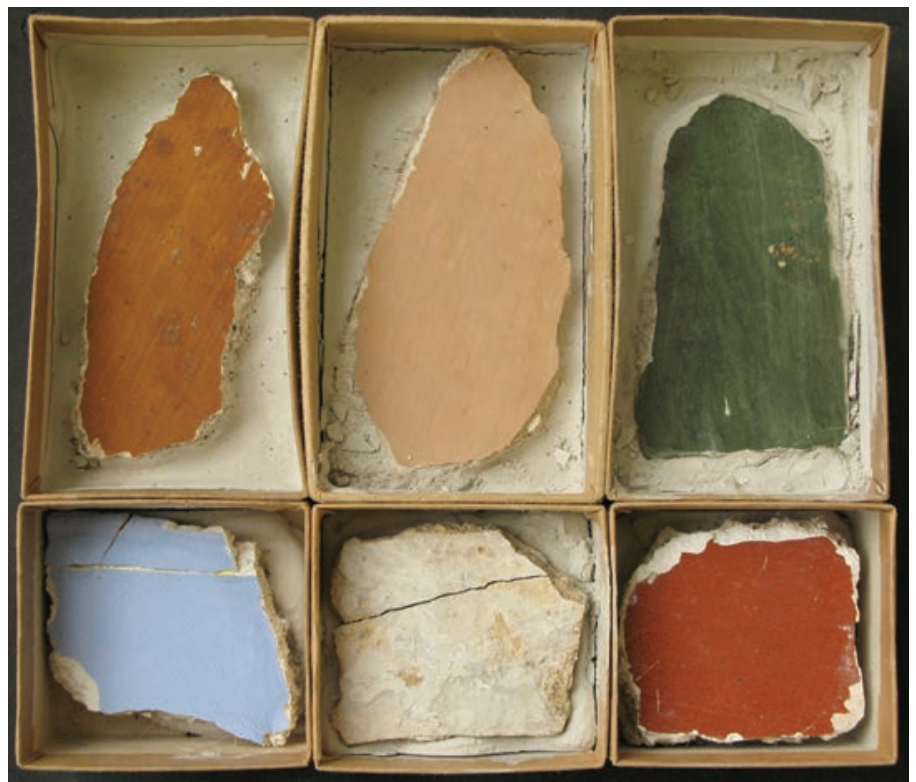

Fragments de murs plâtrés avec leur finition d'origine provenant de différents sites :

a-b-c) Église Christ Church, Sorel ; d) Chapelle anglicane [plafond], Grosse-Île ;

e) Château Saint-Louis [caves], Québec ; f) Eglise Saint-Antoine-de-Bienville, Lévis 


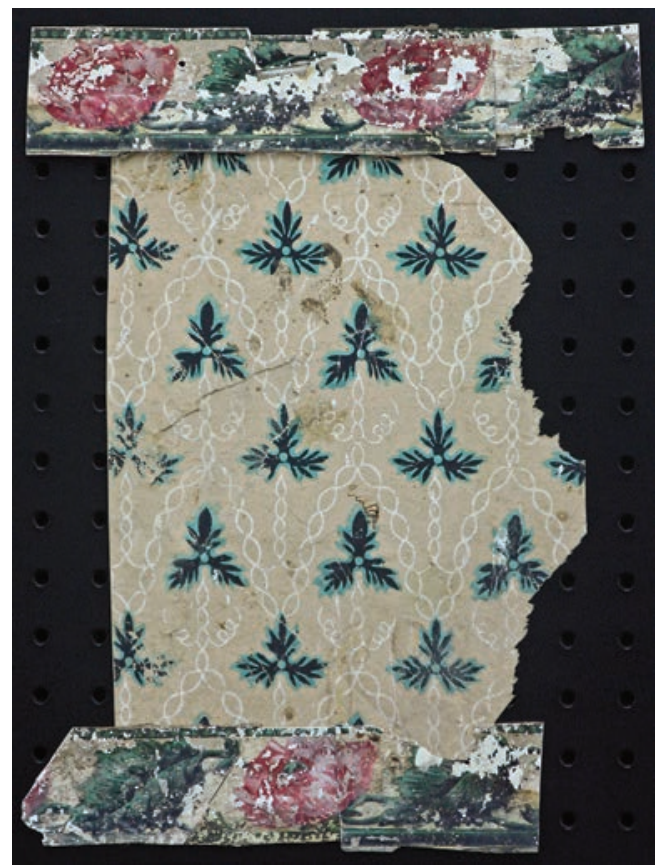

Fragment de papier peint et bordure [v. 1815]

Maison des Jésuites-de-Sillery (Québec)

Photo : Raynald Bilodeau, 2009

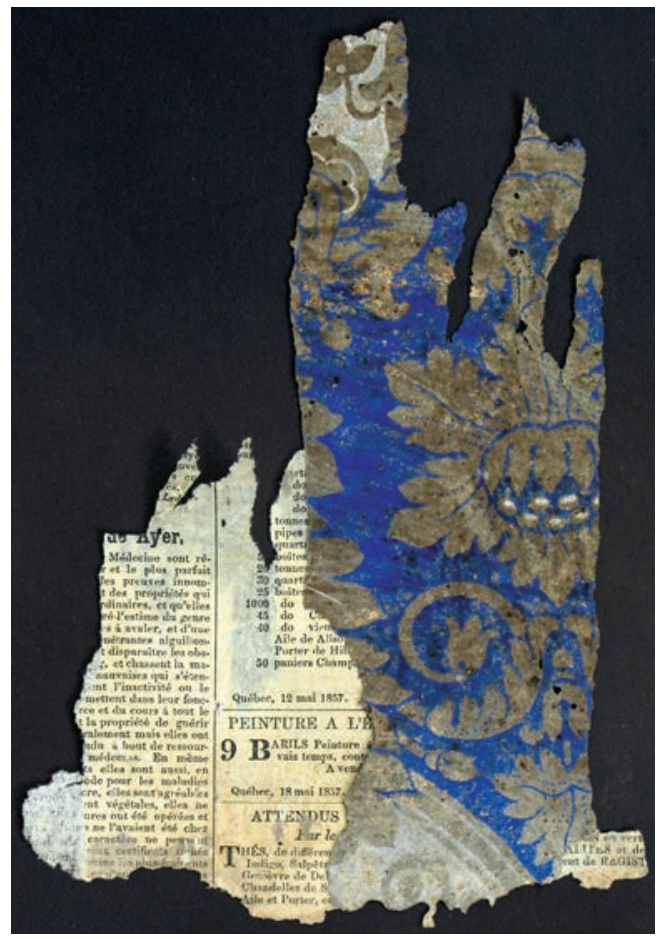

Fragment de papier peint sur son support de papier [journal daté du 12 mai 1857 et 18 mai 1857]

Manoir Mauvide-Genest, Saint-Jean, Île d'Orléans Photo : Raynald Bilodeau, 2008 


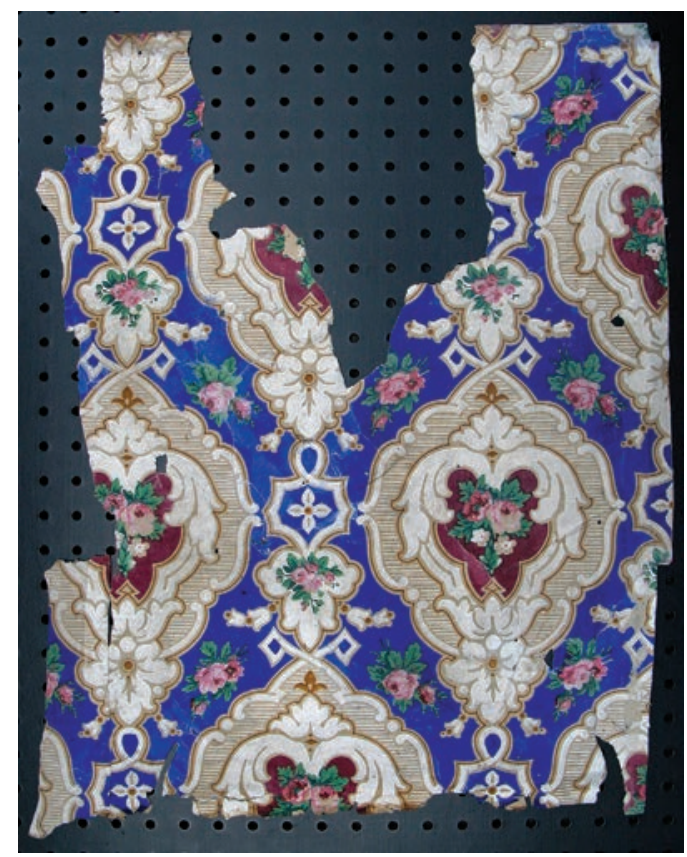

Fragment de papier peint [v. 1855-1860]

Saint-Michel-de-Bellechasse

Photo : Raynald Bilodeau, 2017

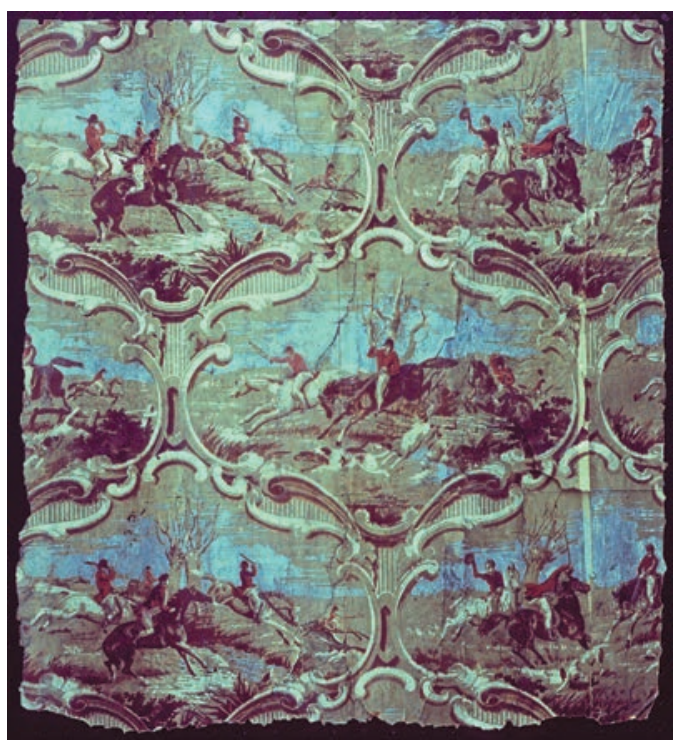

Fragment de papier peint [v. 1860-1865]

Maison Tremblay-Patterson, Beauport

Photo : Raynald Bilodeau, 2009 
Cartier (Montréal), Grosse-Île (plusieurs des bâtiments), le manoir Papineau (Montebello), le manoir Le Boutillier (Gaspé), la Citadelle de Québec, le Fort Saint-Jean, l'Ancien-Édifice-de-la-Douane-de-Québec, le Manège militaire de Québec (après l'incendie), le château De Ramezay (Montréal), la maison et le magasin général Louis-S.-Saint-Laurent (Compton), la maison Hector-Authier (Amos), la maison-Chapais (Saint-Denis-De La Bouteillerie), le DomaineJoly-de-Lotbinière (Sainte-Croix), ou encore la maison Homestead du chantier maritime A.C. Davie (Lévis). Pendant longtemps, les échanges de services entre fonctionnaires du gouvernement du Canada et ceux du gouvernement du Québec n'étaient pas chose courante. En dépit de ce frein, l'expertise de Raynald a souvent été sollicitée par les spécialistes du patrimoine du ministère de la Culture du Québec. Le plus souvent, ces «prêts de services » étaient faits de façon non officielle, échappant en cela aux règles administratives. Mais, comme le dit Raynald, « après tout, c'est le même patrimoine ! » Répondant la plupart du temps à des demandes d'intervention émanant des architectes du ministère de la Culture - dont faisait partie son ami Roger Picard - il a fait de nombreuses interventions sur des sites inscrits au répertoire du patrimoine culturel du Québec, dont l'édifice de la Cour-de-Circuit-de-L'Île-Verte (Île-Verte), la maison René-Lévesque (New-Carlisle), la maison ThéodoreJean-Lamontagne (Sainte-Anne-des-Monts), la maison Déry (Pont-Rouge), le village historique de Val-Jalbert (Chambord), la chapelle de procession Notre-Dame-de-Grâce (L'Ange-Gardien). Des corporations et des villes ont aussi sollicité son expertise ; mentionnons le Centre canadien d'architecture pour une analyse du décor intérieur de la maison Shaughnessy (Montréal), ou en maintes occasions, la Ville de Lévis (à la Terrasse-du-Chevalier-de-Lévis, à la maison natale de Louis Fréchette et à la maison Alphonse-Desjardins). L'expertise de Raynald Bilodeau a contribué à faire avancer la connaissance en matière de finis, faux-finis, peintures et papiers peints, un aspect souvent négligé dans le processus de restauration des bâtiments patrimoniaux.

\section{La collection}

C'est dans le cadre de ces travaux de curetage que Raynald fut amené à s'intéresser de façon toute particulière aux papiers peints. Sa curiosité le poussa à toujours en savoir davantage, à approfondir ses connaissances. Souvent, il se disait : « Il y a d'autres choses que tu ne sais pas, il faut découvrir ce qu'il y a en dessous ». Attentif à la moindre craquelure, brèche ou fissure, il se mit à fouiller avec la fine lame d'un scalpel entre les interstices des planches et les couches de plâtre ou de tapisserie qui recouvraient les murs, pour voir ce qui s'y cachait. Il y fit parfois d'étonnantes découvertes : des papiers peints très anciens enfouis sous plusieurs couches de plâtre, de peinture ou 


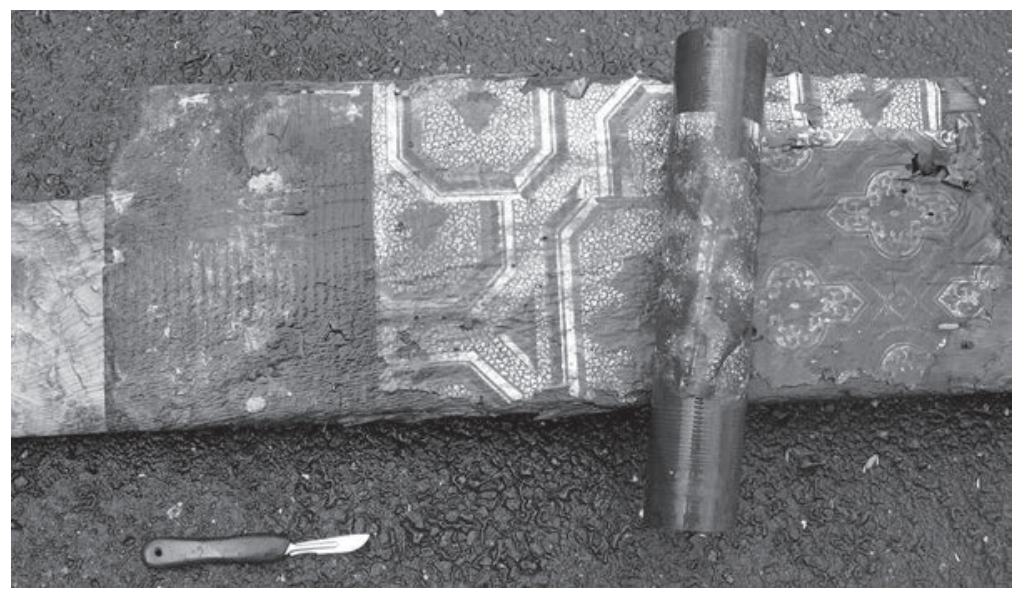

Rouleau de bois permettant le prélèvement de papier peint mouillé très fragilisé. L'outil a été créé par Raynald Bilodeau

Photo : Raynald Bilodeau, 2014

de papier. Dans les maisons modestes, il s'aperçut que le vieux papier peint n'était jamais arraché du mur avant d'en recevoir un nouveau, mais qu'on appliquait plutôt une épaisse couche de papier journal avant d'y apposer le nouveau. En plus d'égaliser et de faire disparaître les imperfections du mur, le papier journal faisait office d'isolant. Il affina son approche et développa une méthode d'investigation - créant même des outils tel ce rouleau de bois vernis qu'il a mis au point pour relever directement sur le terrain ses fragments de papiers peints, parfois des laizes entières - de façon à prélever des échantillons et constituer des dossiers sur chacun des sites expertisés. Sur place, chacun des échantillons est, dans la mesure du possible, détaché en unité, sinon en agrégat. Il lui est même arrivé de récupérer des pans complets de murs (qui autrement étaient expédiés au dépotoir) pour mieux les examiner en laboratoire. En atelier, l'échantillon est séparé de l'agrégat en le faisant tremper dans l'eau. Les couleurs de certains papiers peints étant parfois solubles, elles peuvent facilement se dégrader. Avec l'expérience, le spécialiste a appris à distinguer les papiers peints solubles à l'eau de ceux qui ne le sont pas. Pour certains, il suffit de les humidifier. Le plus souvent, une couche de papier journal sépare chaque séquence. Ces couches de papier journal sont précieuses, car elles sont source d'informations. Elles précisent l'époque, parfois l'année, le mois même où le papier peint a été appliqué sur un mur. Chaque échantillon fait l'objet d'une mise en contexte, d'une localisation, d'une datation, avant d'être inséré entre des cartons pour être ensuite mis à sécher lentement avant d'être rassemblé dans des cahiers. Après avoir 


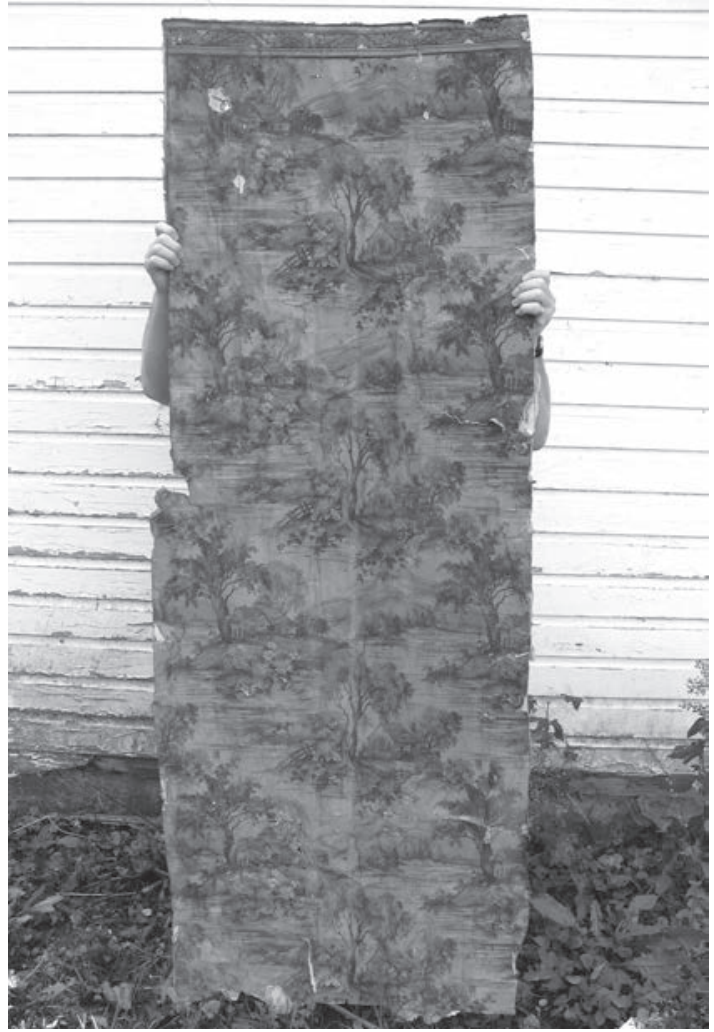

Planches de bois recouvertes de douze couches de papiers peints

Maison Louis-Fréchette, Lévis

Photo : Raynald Bilodeau, 2014

pris sa retraite de Parcs Canada, Raynald se servira plutôt de plastiques au lieu de cartons, plus précisément du « coroplast », ce matériau utilisé dans la fabrication des affiches électorales. L'ensemble de ces cahiers constitue une véritable banque de référence pour l'histoire du papier peint au Québec. On y trouve des informations relatives à la datation des papiers peints, aux provenances, aux influences stylistiques, aux modes de distribution, au milieu social auquel appartenaient leurs propriétaires. Raynald raconte une anecdote qui illustre bien comment, même à partir de minuscules fragments, il peut faire parler les papiers peints. À Compton, dans l'entrepôt du magasin général Louis-S.-Saint-Laurent, un archéologue avait remarqué quelques fragments de papiers chiffonnés mêlés à des éclats de verre entre les murs. À sa demande, Raynald les avait examinés et s'était aperçu qu'il s'agissait de morceaux de papiers peints provenant de rouleaux que des rongeurs avaient déchiquetés. 


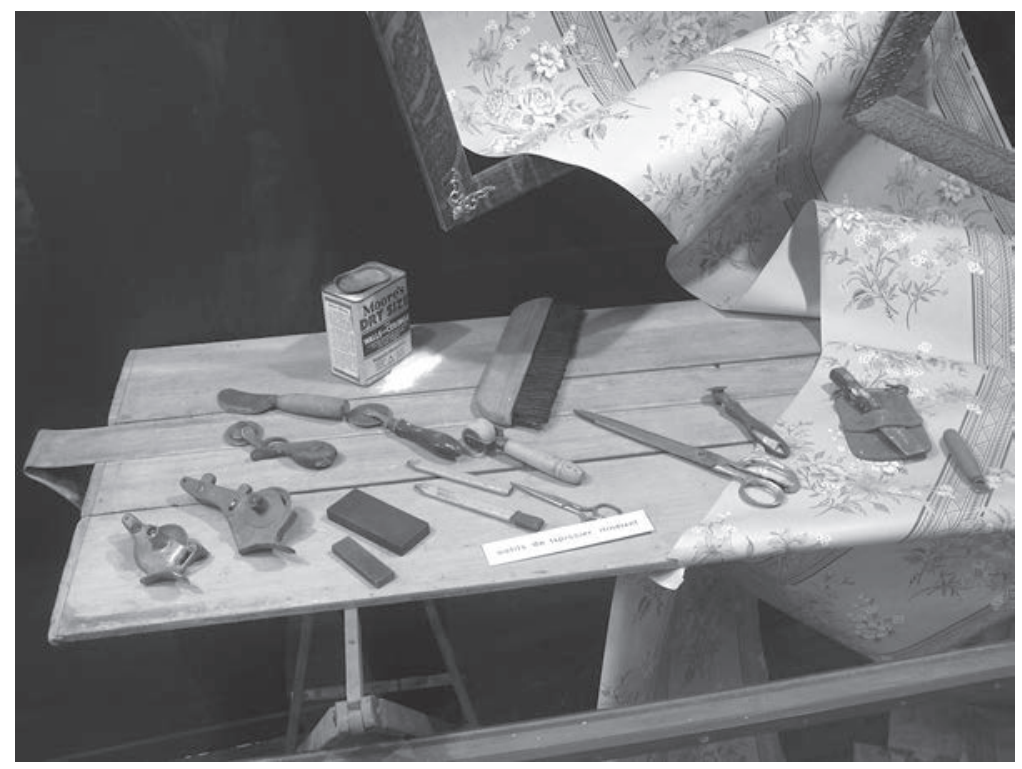

Installation « Outils de tapissier itinérant »

Musée François-Pilote, La Pocatière

Photo : Raynald Bilofeau, 2012

Les morceaux de verre provenaient de globes de lampes à l'huile qu'on avait brisés pour bloquer le passage des rongeurs dans les interstices. Le plus étonnant, c'est qu'à partir de ces minuscules échantillons, le spécialiste avait réussi à recréer leurs motifs et à les dater (vers 1865).

$\mathrm{Au}$ cours des ans et de ses déplacements à travers le Québec, Raynald Bilodeau a ainsi constitué une collection exceptionnelle de papiers peints et de documents (catalogues, brochures), de même que d'outils associés à la pose de papier peint et la réalisation de faux-finis. La collection, constituée entre 1980 et 2010, compte des milliers de fragments de papiers peints regroupant quelque 1200 modèles différents. Ces modèles couvrent l'ensemble du $\mathrm{XIX}^{\mathrm{e}}$ siècle ainsi que les premières décennies du $\mathrm{XX}^{\mathrm{e}}$ siècle. Prélevés directement sur les lieux, au hasard des déplacements du chercheur, ces fragments proviennent d'environ 200 sites différents, tous situés au Québec.

Chacun des sites a été identifié et chaque échantillon a été archivé et souvent daté. Certains de ces sites sont aujourd'hui disparus, soit parce qu'ils ont été incendiés, soit parce qu'ils ont été démolis. L'ensemble de la documentation est rassemblé dans des cartables selon l'ordre chronologique de la collecte. Ces papiers peints proviennent aussi bien de milieux modestes que 


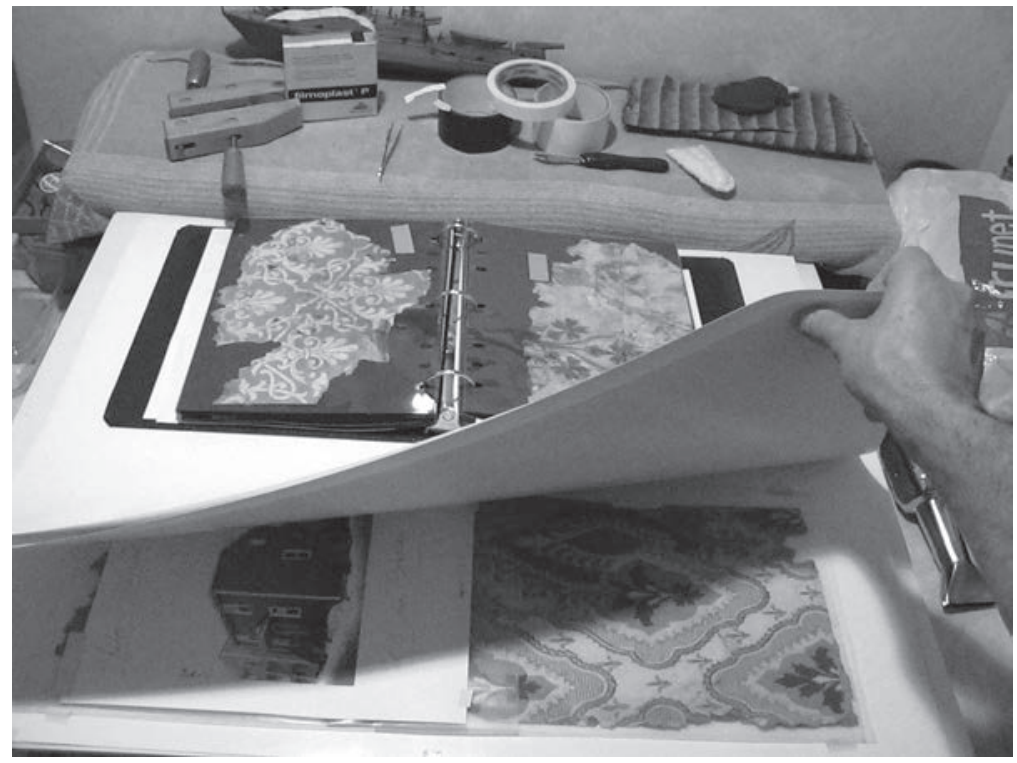

Reconstitution des couches successives et catalogage des données Photo : Raynald Bilodeau, 2015

de milieux plus aisés. Les papiers de grande qualité, pour la plupart importés d'Europe, étaient considérés comme des éléments de décor luxueux. Étant donné leur nature ils se sont bien conservés, ce qui n'est pas le cas des papiers bons marchés fabriqués mécaniquement à partir des années 1840. Ceux-ci se détérioraient rapidement et étaient remplacés fréquemment, ce qui explique pourquoi dans une même pièce on pouvait en superposer jusqu'à quinze couches d'épaisseur.

En plus de ces milliers de fragments de papiers peints, la collection comprend 55 catalogues d'échantillons provenant de différents fabricants et distributeurs canadiens, tels The National Wall Paper Co. Ltd, Colin Mc Arthur \& Co. Ltd, Watson, Foster Co. Ltd, Eaton's Walpapers Ltd, et plusieurs autres. Les plus anciens de ces catalogues datent de 1915, les plus récents de 1970; avant le Xx $x^{\mathrm{e}}$ siècle, il n'y avait pas de catalogue pour le papier peint. Ces catalogues étaient distribués partout au Québec par l'intermédiaire de représentants, le magasin général servant le plus souvent de point de vente en région. La collection comprend également une dizaine de brochures et de cartes publicitaires de manufacturiers de papiers peints du début du $\mathrm{xx}^{\mathrm{e}}$ siècle (1902-1923). Quant aux outils, il s'agit de 21 instruments qui 


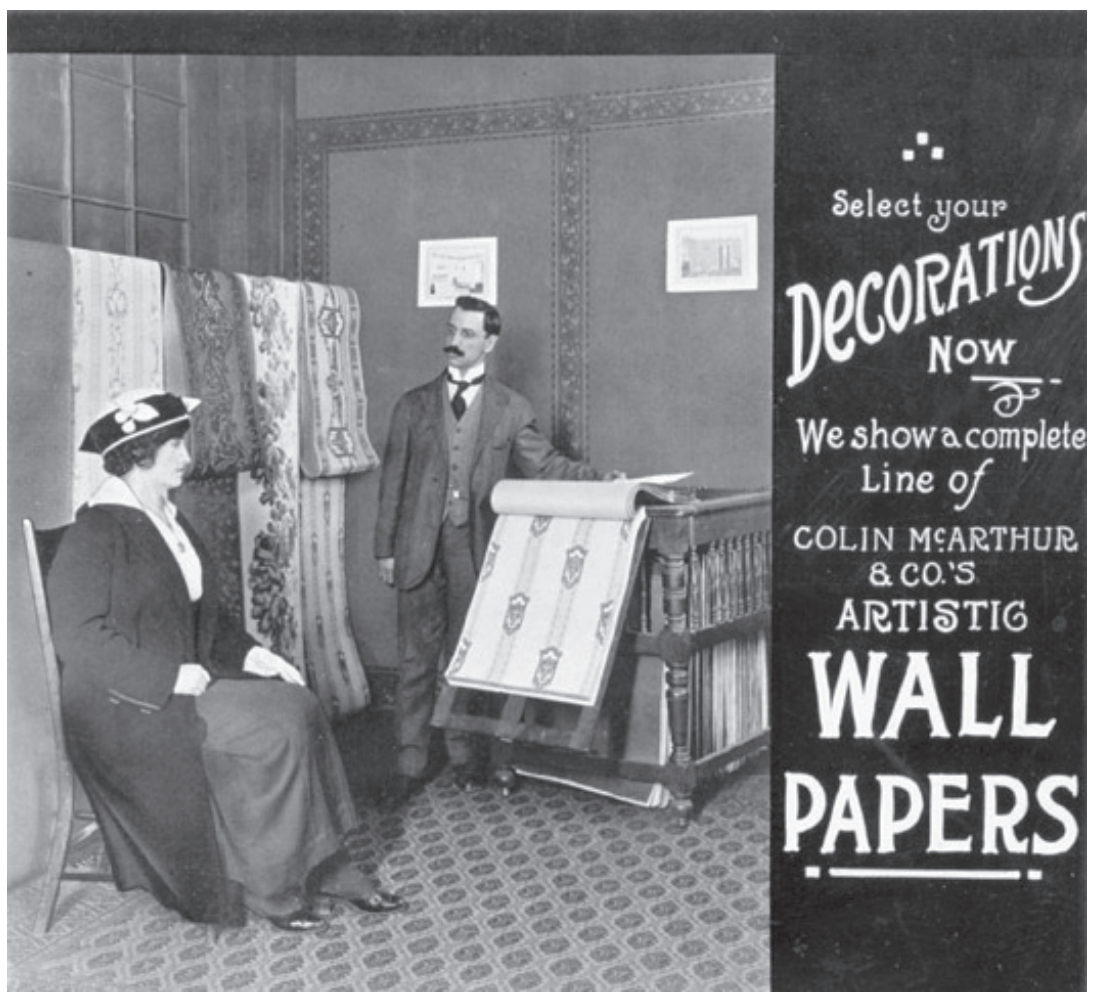

Un vendeur présente un modèle de papier peint à une cliente

Réclame publicitaire de la maison Colin Mc Arthur \& $\mathrm{C}^{\circ}$., fabricant et distributeur de papiers peints, Montréal, vers 1912

Coll. Raynald Bilodeau

servaient à la pose du papier peint et à la réalisation de faux-finis. Ces instruments proviennent de la région du Richelieu et remontent au début du $\mathrm{XX}^{\mathrm{e}}$ siècle.

La collection constitue un outil de référence incontournable, notamment dans le cadre de travaux de restauration et pour l'interprétation des intérieurs de lieux historiques. C'est aussi une source inestimable pour la connaissance de l'histoire de la vie quotidienne au Québec, car elle permet d'évoquer plusieurs aspects de l'évolution des goûts et des usages dans le décor de l'habitat domestique québécois entre 1800 et 1930, en plus de témoigner des grands courants stylistiques et des habitudes de vie des occupants. Acquise par Parcs Canada en 2011, la collection constitue une contribution importante à l'histoire de la vie domestique au Québec. C'est d'ailleurs la seule collection de ce genre connue au Québec. En soi, le processus de curetage des finis de bâtiments était une pratique relativement courante depuis le milieu du 


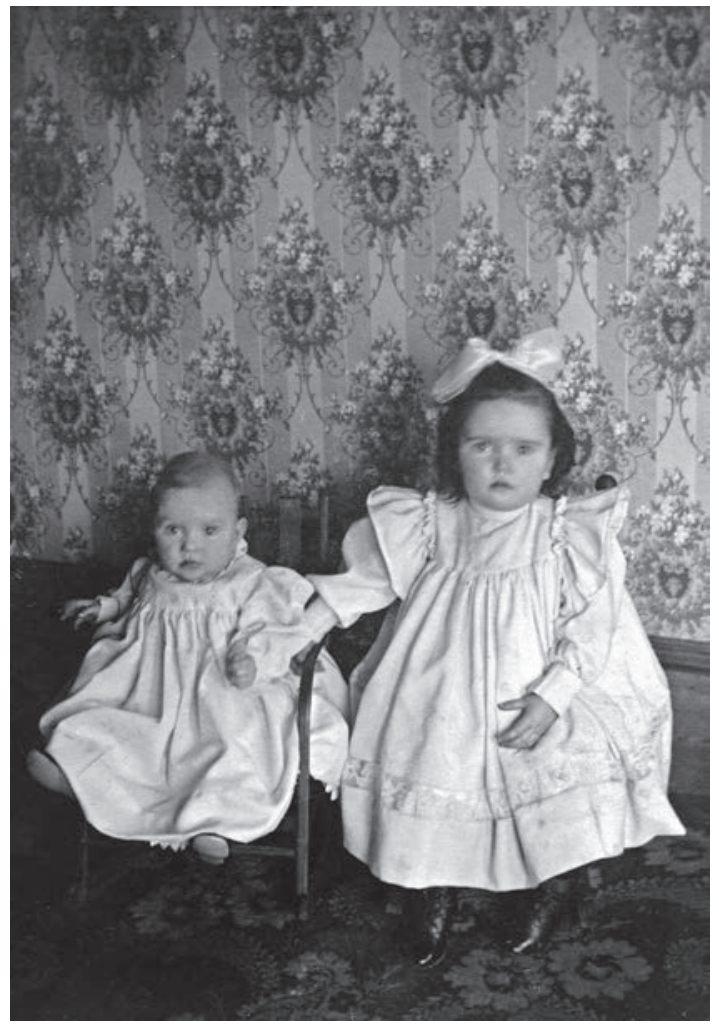

Photographie hors studio de jeunes enfants devant un mur recouvert de papier peint, vers 1900

Coll. Raynald Bilodeau

$\mathrm{Xx}^{\mathrm{e}}$ siècle, mais pas ici. Au Québec, Raynald Bilodeau est le premier à avoir introduit cette pratique et à avoir développé une méthode pour le relevé et l'analyse des finis sur les bâtiments anciens. Si Parcs Canada fait figure de leader dans le domaine, c'est beaucoup grâce à son initiative.

\section{Une retraite active}

Raynald Bilodeau a pris sa retraite de Parcs Canada en 2011 après plus de 30 ans de service, mais il n'a pas pour autant cessé ses activités de chercheur et collectionneur ; il continue son travail d'investigateur sur le monde qui l'entoure. Dans le prolongement de ses activités professionnelles, il s'adonne à deux occupations qui relèvent de son besoin de créer et le pousse à « aller voir derrière les apparences ». En photo, ce qui l'intéresse, c'est de porter un regard inédit sur les empreintes laissées par l'homme sur son environnement, là où les éléments naturels et le passage du temps font se rencontrer, dans un 
amalgame souvent inattendu, objets naturels et artéfacts. C'est, dit-il, l'ethnologue en lui qui l'amène à chercher dans la nature les traces de l'activité humaine. C'est aussi ce qu'il fait dans l'exercice d'une forme d'art populaire qu'il pratique depuis de nombreuses années avec sa conjointe, Lise Cardinal, et qu'il qualifie d' " Art-Efact» ou encore - selon une formule empruntée à une tradition des landes de la Gascogne - d' " art de la coustille ». La « coustille » est une tradition qui consiste à récupérer ce que l'océan rejette sur la plage les lendemains de grosse houle. À Vieux-Boucau (région Nouvelle-Aquitaine), on en a fait une fête, celle « de la coustille et du bois flotté ». Depuis des années, Raynald et sa compagne arpentent les rives du Saint-Laurent particulièrement sur la Côte-du-Sud, car ils habitent Lévis - à la recherche de bois de grève et autres matériaux (tessons de verre, morceaux de porcelaine et de métal) dont ils se servent pour créer des objets d'art, des sculptures et des lampes, des «Lum-hier » comme il les désigne. Raynald documente chacune de ses productions en les photographiant et, par déformation professionnelle, monte des dossiers sur chacune d'elles. Ces dossiers incluent des photos de toutes les pièces qui composent l'œuvre.

Raynald n'a jamais non plus cessé de s'intéresser aux papiers peints. Il poursuit ses recherches sur le terrain. Ses connaissances et son expertise sont encore fréquemment sollicitées et il n'accepte d'intervenir à titre d'expert conseil ou de conférencier que dans son milieu d'appartenance (Lévis et les environs), souvent bénévolement. Travailleur de l'ombre pendant la plus grande partie de sa vie, sa plus grande satisfaction est d'avoir convaincu plusieurs intervenants du milieu du patrimoine de l'importance de porter attention aux décors extérieurs et intérieurs des bâtiments patrimoniaux avant d'entreprendre des travaux de rénovation et de restauration.

\section{Prix et distinctions}

En lien avec ses trente années de carrière à Parcs Canada, Raynald Bilodeau a reçu de nombreuses reconnaissances de son employeur et, notamment, une nomination dans le cadre du Prix du patrimoine de Lévis, catégorie interprétation et diffusion (2015). Entre 1972 et 2017, en photographie, Raynald Bilodeau a reçu pas moins d'une trentaine de prix dont : deuxième prix au Salon de la Société des photographes artisans du Québec (1979); grand prix du $38^{\mathrm{e}}$ Salon des photographes artisans du Québec (1980); deuxième prix canadien de la Fondation Héritage Canada (1983); gagnant de deux premiers prix de la revue France-Nord (1991); gagnant d'un premier prix de la revue France-Vert (1992). 